\title{
Hybrid Synchronization Scheme for Multi-Carrier Communication Systems
}

\author{
Eung Sun $\mathrm{Kim}^{1} \cdot$ Sang Kyu Park $^{2}$
}

\begin{abstract}
In this paper, we develop a symbol/frame time and carrier frequency synchronization scheme for multi-carrier signaling in wireless mobile channels. The proposed scheme achieves simultaneous time synchronization and carrier frequency offset estimation. Simulation results show that the frequency offset of multiple sub-carrier spacings can be estimated and that performance is improved with robustness regardless of the cyclic prefix length.
\end{abstract}

Key words: Carrier Frequency Offset, OFDM, Preamble, Synchronization, Timing Metric.

\section{Introduction}

Time/frequency synchronization is a key factor for the performance of multi-carrier systems. Landström uses a cyclic prefix synchronization method that is effective because there is no redundancy in transmissions [1]. However, the estimation range of the carrier frequency offset is less than half of the sub-carrier spacing. Schmidl proposed a pilot symbol pattern which can also be used as a preamble for synchronizations [2]. Nevertheless, the time synchronization error is great because of the plateau of the timing metric during the cyclic prefix duration. Minn proposed a pilot symbol technique, which is a modified version of Schmidl's method, where a carrier frequency offset over two sub-carrier spacings cannot be estimated [3].

The design of a pilot symbol similar to Minn's method is used in wireless LAN systems such as IEEE 802.11a [4]. In conventional schemes, multi-path fading or cyclic prefix length causes severe time synchronization errors and the frequency offset of multiple sub-carrier spacings cannot be estimated. Therefore, we propose a novel algorithm that is robust against multi-path fading and can estimate the carrier frequency offset of multiple sub-carrier spacings. The proposed preamble has a repetitive structure of two different pilot patterns with differ- ent lengths. Based on our proposed scheme, both time and frequency synchronizations are acquired simultaneously in a multi-carrier symbol duration.

\section{II . System Model}

A baseband signal for multi-carrier systems is generated by inverse fast Fourier transform (IFFT) of complex data symbols. They are parallel-to-serial converted and then up-converted to radio frequency (RF) signal. The baseband transmit signal is given by

$$
s(n)=\sum_{m=0}^{N-1} C_{m} \exp \left(j \frac{2 \pi m n}{N}\right)
$$

where $C_{m}$ is a complex valued symbol on the m-th sub-carrier, $N$ is the number of the IFFT points. The received signal is affected by the carrier frequency offset, $f_{\text {offset }}$, between the transmitter and the receiver oscillator. The timing offset, $D$, in additive white Gaussian noise (AWGN) can be expressed as

$$
r(n)=s(n-D) \exp (j \cdot 2 \pi \cdot \delta f \cdot n)+w(n)
$$

where $\delta f$ is the phase change for a sample time duration, and $w(n)$ is AWGN. The received signal is downconverted to a baseband signal and then demodulated.

\footnotetext{
Manuscript received May 7, 2012 ; Revised June 26, 2012 ; Accepted August 13, 2012. (ID No. 20120507-013J)

${ }^{1}$ Dept. of Electronics \& Computer Engineering, Hanyang University, Seoul, Korea.

Samsung, Signal \& Systems Lab., Samsung Advanced Institute of Technology (SAIT), Yongin, Korea.

${ }^{2}$ Dept. of Electronics \& Computer Engineering, Hanyang University, Seoul, Korea.

Corresponding Author : Sang Kyu Park (e-mail : skpark@hanyang.ac.kr)
}

This is an Open-Access article distributed under the terms of the Creative Commons Attribution Non-Commercial License (http://creativecommons.org/licenses/ by-nc/3.0) which permits unrestricted non-commercial use, distribution, and reproduction in any medium, provided the original work is properly cited. 


\section{Synchronization Method}

We propose the preamble structure as

$$
P_{\text {prop }}=\left[\begin{array}{llll}
A_{\text {prop }} & A_{\text {prop }} & B_{\text {prop }} & B_{\text {prop }}
\end{array}\right]
$$

where $A$ and $B$ represent the $N_{a}$ and $N_{b}$ length samples, respectively. The lengths of $A$ and $B$ are different, and the preamble size is $N$, where $2 N_{a}+2 N_{b}=N$. The symbol time recovery is performed using the following timing metric:

$$
\begin{aligned}
& M(n)=\frac{|P(n)|^{2}}{(R(n))^{2}} \\
& P(n)=a b s\left(\sum_{k=0}^{N_{a}-1} r^{*}(n+k) r\left(n+k+N_{a}\right)\right)+ \\
& a b s\left(\sum_{k=0}^{N_{b}-1} r^{*}\left(n+k+2 N_{a}\right) r\left(n+k+N_{b}+2 N_{a}\right)\right) \\
& R(n)=\sum_{k=0}^{N_{a}-1}\left|r\left(n+k+N_{a}\right)\right|^{2}+\sum_{k=0}^{N_{b}-1}\left|r\left(n+k+N_{b}+2 N_{a}\right)\right|^{2}
\end{aligned}
$$

The start of symbol time is found using a maximum of the timing metric. The carrier frequency offset is also estimated by using a short preamble pattern. The estimation range of the carrier frequency offset can be expanded by changing the length of short preamble pattern, where the length of $\mathrm{A}$ is assumed to be larger than B. The receiver correlation of the short preamble pattern at the correct symbol time is given by

$$
P_{f}(D)=\sum_{k=0}^{N_{b}-1} r^{*}\left(D+k+2 N_{a}\right) r\left(D+k+N_{b}+2 N_{a}\right)
$$

The carrier frequency offset is estimated by using the phase of $P_{f}(D)$.

$$
f_{o f f s e t}=\frac{\Delta f}{2 \pi} \frac{N}{N_{b}} \cdot \angle P_{f}(D)
$$

We can estimate the carrier frequency offset smaller than $\frac{1}{2} \frac{N}{N_{b}} \Delta f$. The proposed algorithm works well even when the carrier frequency offset is multiple sub-carrier spacings. Schmidl proposed a preamble structure that repeats a $N / 2$ length pilot pattern [2]. Minn and IEEE802.11a use a preamble structure that repeats a $N / 4$ length pilot pattern [3], [4]. In [2], the timing metric has a plateau because the cyclic prefix is a copy of a multicarrier symbol, which leads to incorrect time synchronization. It can only estimate the carrier frequency offset smaller than one sub-carrier spacing. In [3] and [4], the timing metric has a plateau or two peaks, according to the cyclic prefix length. This also causes a time synchronization error, where only a carrier frequency offset that is smaller than two sub-carrier spacings can be estimated.

\section{Conclusion}

The performance of the proposed method is evaluated by computer simulations. Performance comparison of the time synchronization and carrier frequency offset synchronization are investigated for four cases: 1) Schmidl's method; 2) Minn's method; 3) IEEE802.11a; and 4) the proposed method.

The simulation environments are as follows: the bandwidth is $20 \mathrm{MHz}$, the number of sub-carriers is 1024 , and the number of cyclic prefix is 128 or 256 . We assume that $N_{a}$ is greater than $N_{b}$. Here, $N_{a}$ and $N_{b}$ are 384 and 128, respectively. Fig. 1 and Fig. 2 show the timing metric when the cyclic prefix length is 128 and 256, respectively. In both figures, the proposed scheme is observed to have one peak at the accurate symbol time, independent of the ratio of the cyclic prefix length to the multi-carrier symbol length. However, the other methods show plateaus or additional peaks, which depend on the cyclic prefix length.

Fig. 3 and Fig. 4 show the mean of the carrier frequency offset estimation results when the offset is one sub-carrier spacing and three sub-carrier spacings, respectively. We consider the outdoor to indoor and pedestrian channel B that is recommended by the International Telecommunication Union (ITU). The proposed method finds the carrier frequency offset, exactly even

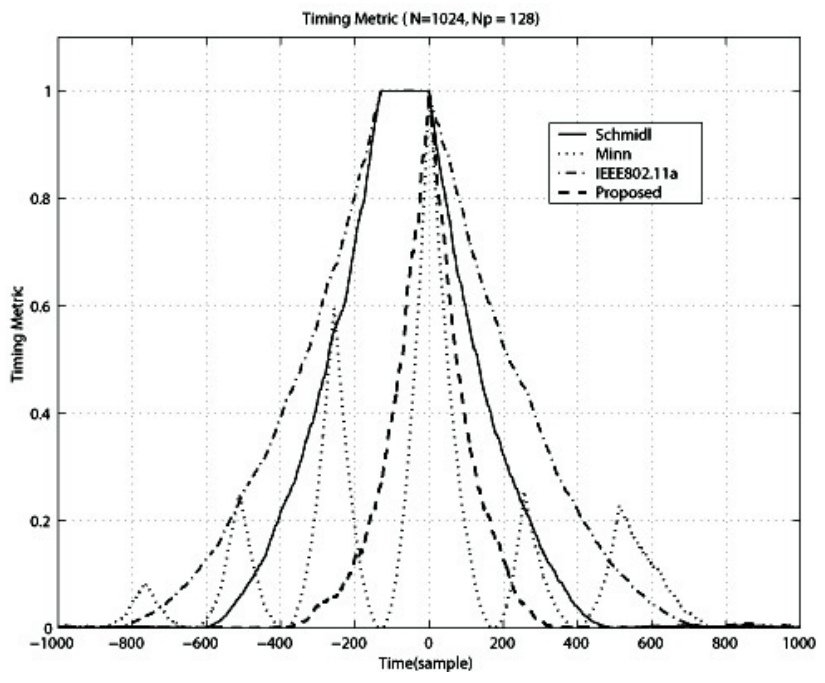

Fig. 1. Timing metric $\left(N=1024, N_{c p}=128\right)$. 


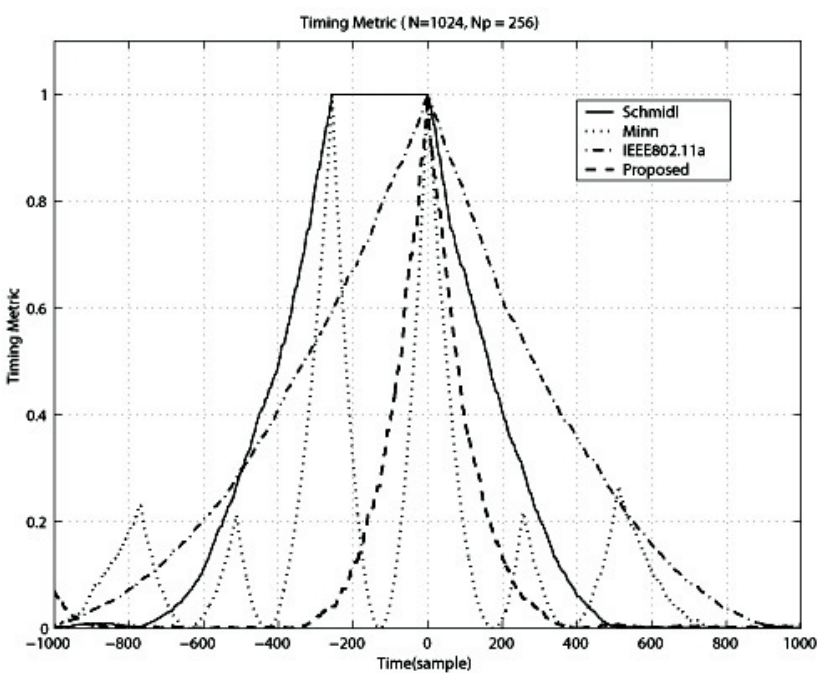

Fig. 2. Timing metric $\left(N=1024, N_{c p}=256\right)$.

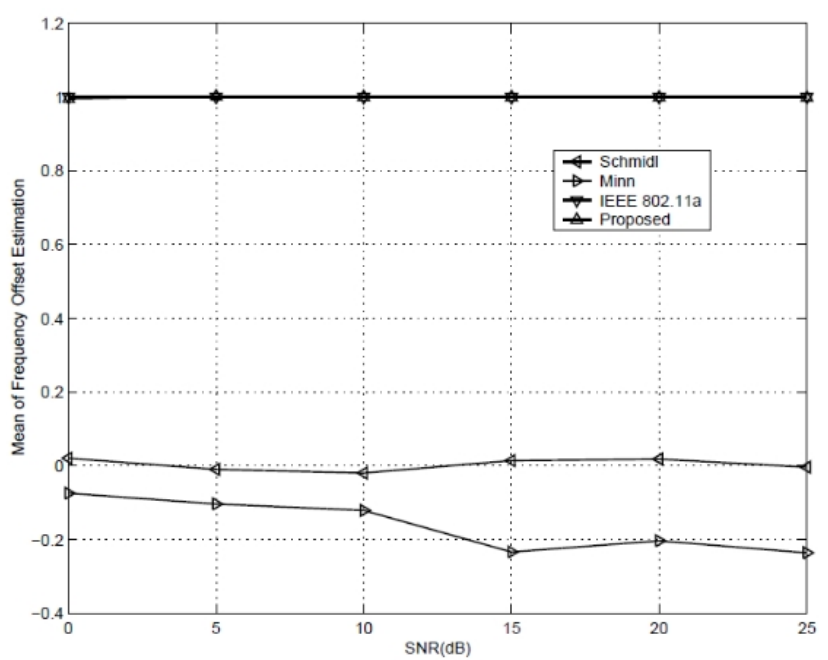

Fig. 3. Mean of carrier frequency offset estimation (carrier frequency offset is one sub-carrier spacing).

with low SNRs and severe multi-path environments, while other methods cannot estimate the carrier frequency offset because their carrier frequency offset estimation range is less than two sub-carrier spacings.

The proposed method finds the correct symbol time regardless of the cyclic prefix length, and estimates carrier frequency offsets of multiple sub-carrier spacings.

\section{Discussion}

We described a synchronization method and compared the performance of different algorithms. We proposed a novel preamble structure and receiver algorithm for time

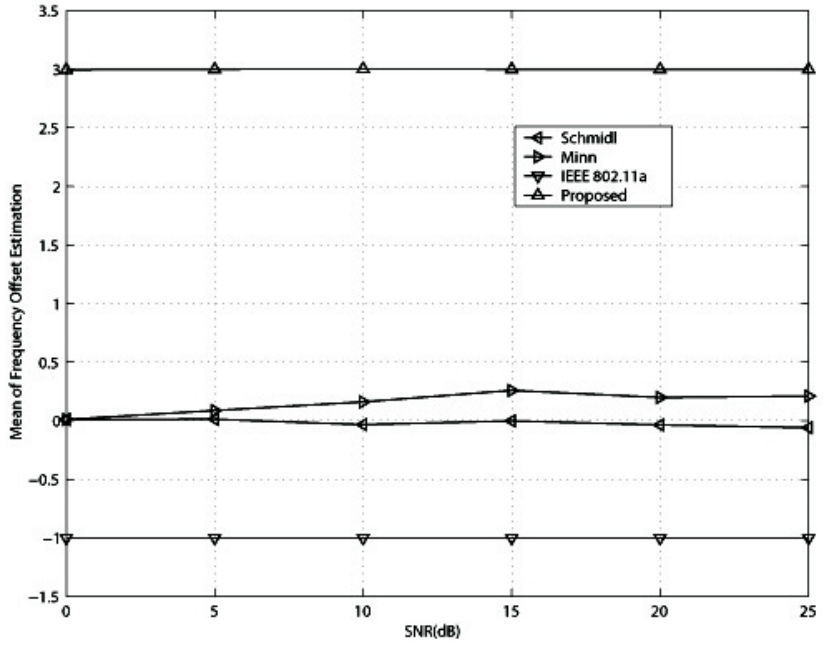

Fig. 4. Mean of carrier frequency offset estimation (carrier frequency offset is three sub-carrier spacings).

synchronization and carrier frequency offset estimation that is suitable for future wireless communication systems. The synchronization in a multi-carrier symbol duration was performed using the proposed preamble structure. The estimation range of the carrier frequency offset is expanded by changing the pilot pattern length and the performance of time synchronization; this becomes reliable regardless of cyclic prefix length. The proposed algorithm is shown to be robust against severe multi-path environments.

\section{References}

[1] D. Landström, S. K. Wilson, J. -J. van de Beek, P. Ödling, and P. O. Börjesson, "Symbol time offset estimation in coherent OFDM systems," IEEE Transactions on Communications, vol. 50, no. 4, pp. 545549, Apr. 2002.

[2] T. M. Schmidl, D. C. Cox, "Robust frequency and timing synchronization for OFDM," IEEE Transactions on Communications, vol. 45, no. 12, pp. 16131621, Dec. 1997.

[3] H. Minn, M. Zeng, and V. K. Bhargava, "On timing offset estimation for OFDM systems," IEEE Communications Letters, vol. 4, no. 7, pp. 242-244, Jul. 2000.

[4] IEEE Std 802.11a-1999 Part11: Wirelss LAN Medium Access Control (MAC) and Physical Layer (PHY) Specifications: High-Speed Physical Layer in the 5 GHz Band. 